\title{
Total physical activity and the risk of breast cancer among Nigerian women
}

Samuel O Azubuike, Louise Hayes, Richard McNally

Institute of Health \& Society, Newcastle University

\section{Purpose}

Breast cancer incidence in Nigeria has risen by $>120 \%$ since 2010. Its mortality rate (25.9/100 000/year) ranks highest in Africa. Though Physical inactivity has been linked to increased breast cancer risk in developed countries, its role is yet to be established in sub-Saharan Africa. There is concern that increasing rural-urban migration in Nigeria might be decreasing the population Physical activity (PA) level. The purpose of this study was to investigate if there is an association between physical activity and breast cancer risk among Nigerian women.

\section{Methods}

The study was a multisite hospital based case-control design, involving 379 histologically confirmed breast cancer cases and 403 controls aged $\geq 20$ years. The participants were interviewed in-person between October 2016 and May 2017 using a pretested questionnaire. Total PA was computed by summing occupational, household, transport and recreational PA. All PAs were summarised as both hours per week and metabolic equivalents (MET) hours per week (met-hr/wk). Data analysis was based on Multivariate logistic regression using SPSS version 23.

\section{Results}

The odds of having breast cancer(based on MET-hr/wk) was 38.1\% less among women at the upper tertile of total PA than those at the lowest tertile $(95 \% \mathrm{Cl}: 0.387$, 0.989 ). This was stronger among younger (OR: $0.408,95 \% \mathrm{Cl}: 0.212,0.783$ ) and premenopausal women (OR:0.594, 95\%Cl: 0.195,0.748).

\section{Conclusions}

There was an inverse association between physical activity and breast cancer risk. Regular engagement in variety of physical activity could contribute towards reducing the risk of breast cancer in Nigeria. 